\title{
An Analysis Methodology for the Gamma-ray Large Area Space Telescope
}

\author{
Robin D. Morris* and Johann Cohen-Tanugi ${ }^{\dagger}$ \\ "RIACS, NASA Ames Research Center, MS 269-2, Moffett Field, CA 94035, USA, \\ rdmeemail.arc.nasa.gov \\ †Istituto Nazionale di Fisica Nucleare, Pisa, Italy, johann. cohenepi . infn. it
}

\begin{abstract}
The Large Area Telescope (LAT) instrument on the Gamma Ray Large Area Space Telescope (GLAST) has been designed to detect high-energy gamma rays and determine their direction of incidence and energy. We propose a reconstruction algorithm based on recent advances in statistical methodology. This method, alternative to the standard event analysis inherited from high energy collider physics experiments, incorporates more accurately the physical processes occurring in the detector, and makes full use of the statistical information available. It could thus provide a better estimate of the direction and energy of the primary photon.
\end{abstract}

\section{INTRODUCTION}

Gamma rays are produced by some of the highest energy events in the universe, and their study is critical to understanding the source locations and production mechanisms of ultra-relativistic cosmic particles [1, and refs therein].

The LAT instrument on the Gamma Ray Large Area Space Telescope (GLAST) satellite is scheduled for launch in late 2006. Its goal is to observe the gamma ray sky in the energy range $20 \mathrm{MeV}$ to $300 \mathrm{GeV}$.

At these energies, the photon interacts with matter primarily by annihilating into an electron positron pair. As a consequence, its parameters of interest (direction and energy) can be estimated only through the subsequent interaction of the two charged tracks with the active material of the detector. In the next section we describe in more detail this detection process and emphasize the challenges that it raises. We then outline our analysis methodology and show its application to a simulation of a simplified detector.

\section{THE LARGE AREA TELESCOPE INSTRUMENT}

The Large Area Telescope (LAT) instrument (Figure 1) is designed to determine the energy and direction of gamma ray photons that arrive at the detector. The detector is a stack of conversion and detection layers. The gamma ray photon is converted into an electron/positron pair in one of the conversion layers, and these particles cascade through the detector. The electron and positron are themselves subject to secondary scattering, and also produce further electrons and photons. All these particles trigger the detector strips, which read out the position where a particle crossed the detector 


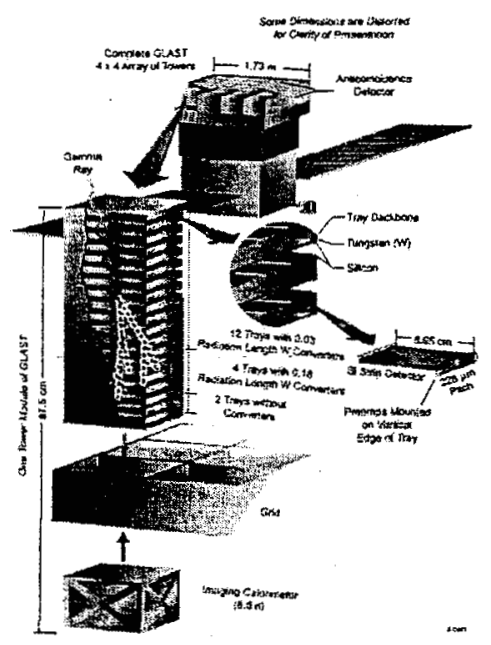

FIGURE 1. Exploded view of the LAT showing an incident gamma ray entering one tower.

layer. The particles finally come to rest in the calorimeter, which measures the remaining energy. The anticoincidence detector eliminates event triggers due to charged cosmic rays (incident charged particles trigger the anticoincidence detector; incident photons do not.) Figure 1 shows a schematic of the LAT. The main output from the detector is the set of positions of the microstrip detectors that were triggered, together with the response of the calorimeter. It is from this set of positions and the calorimeter response that the energy and direction of the photon must be determined.

Figure 2 shows a simulation of a $200 \mathrm{MeV}$ photon arriving at the detector, obtained with the current software written by the GLAST collaboration [2]. The diagonal line extending to the top of the figure (blue) is the incident gamma ray, which and after conversion, splits into the two tracks followed by the electron and positron. It can be seen that the tracks are not straight, indicating significant secondary scattering. The small oblique squares (orange) indicate the microstrip detectors that fired during the event. The small cubes towards the bottom of the figure (blue and red) are the response of the calorimeter. The large rectangles on the right hand side and front (green) are tiles of the anticoincidence detector firing due to the incidence of a secondary electron. The tracks of some of the secondary particles are also shown (black lines). It is clear that most of the firings are due to the passage of the electron/positron pair, but that some are caused by hits from secondary electrons. These firings, together with the significant secondary scattering, represent a challenge for the correct reconstruction of the photon incident direction and energy.

\section{THE PHYSICS OF THE DETECTOR}

The primary physics process is pair production, where a gamma ray is converted into an electron/positron pair. The tungsten foils in the detector are dimensioned such that the conversion probability in a given foil is approximately $2 \%$ (and depends only very 


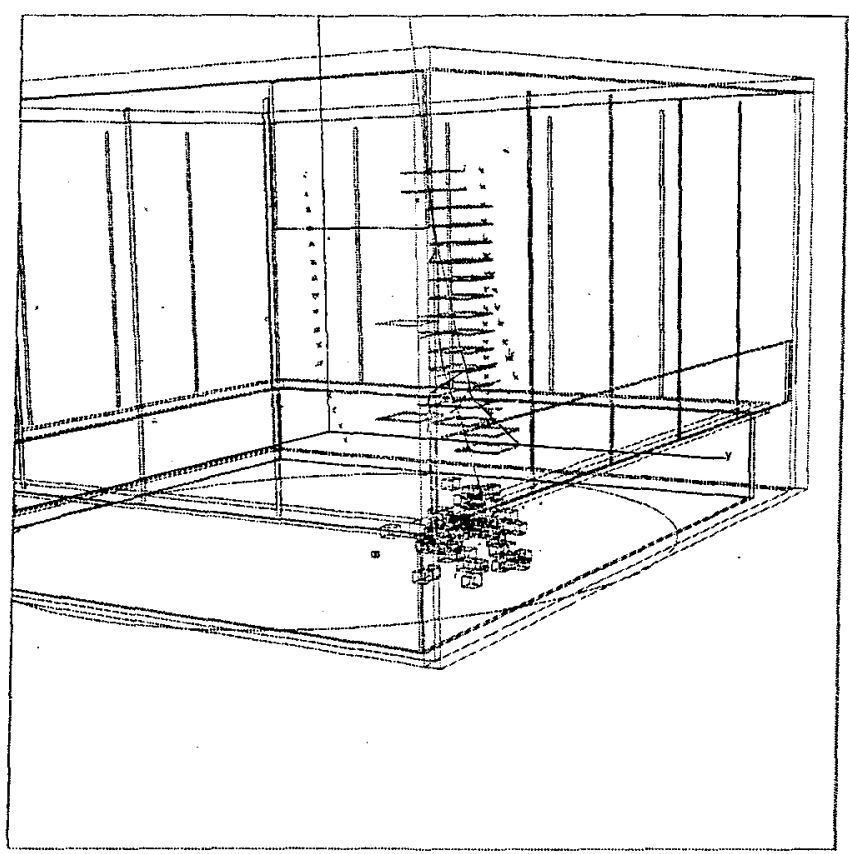

FIGURE 2. Simulation of the interaction of a $200 \mathrm{MeV}$ photon with the LAT.

weakly on energy). This represents a design compromise - thicker foils would give greater conversion probability, but would also increase the effects of multiple scattering and other secondary physical processes which obscure the primary event.

In the conversion event the energy of the incident photon is divided stochastically between the electron and positron. Figure 3 (left) shows the distribution of the energy split as a function of photon energy [3]. Note that there is a reasonable probability that one of the secondary particles will be of very low energy, and thus very susceptible to being scattered or annihilated. The divergence angles of the electron and positron depend stochastically on the energies, as

$$
\phi_{e / p}=\frac{m_{e} c^{2}}{E_{e / p}} u
$$

where $u$ is distributed as shown in figure 3 (right).

The other physics processes that must be taken into account in the event analysis are:

- multiple Coulomb scattering is the cumulative effect of multiple scatters of a charged particle traversing a medium. The name derives from the fact that the main component is the deflection due to Coulomb scattering on the nuclei. If we neglect the tails (corresponding to large scatter), the distribution of the deflection angle projected on a plane is approximately Gaussian, with a width given by:

$$
\theta_{0}=\frac{13.6 \mathrm{MeV}}{\beta c p} z \sqrt{x / X_{0}}\left[1+0.038 \log \frac{x}{X_{0}}\right]
$$



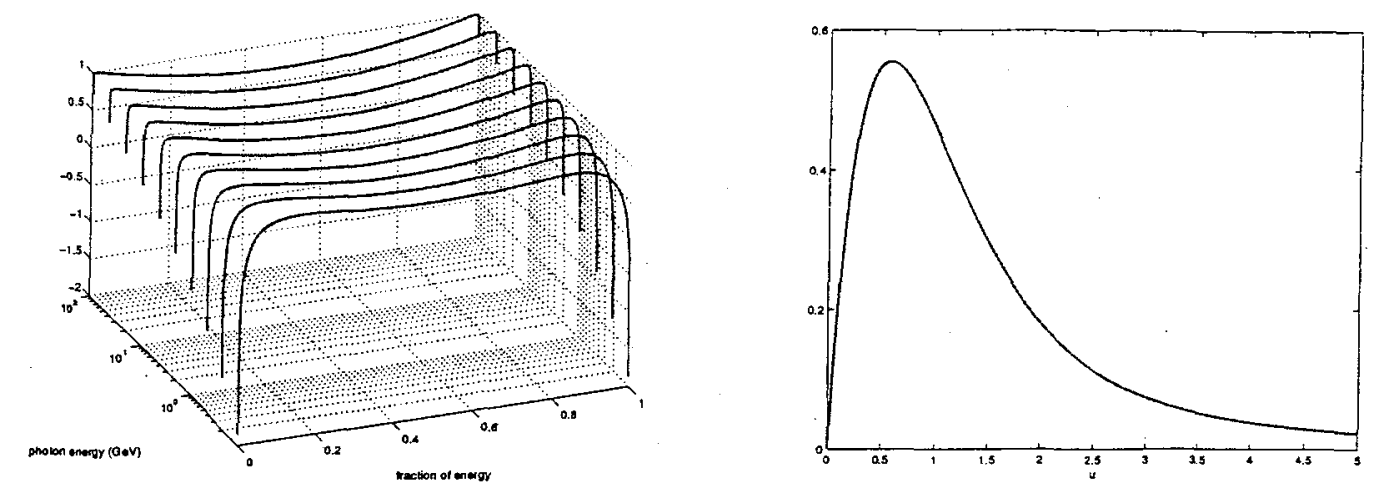

FIGURE 3. Distribution of the energy split between the electron and positron (left). Distribution of the auxiliary quantity in determining the electron/positron angles (right).

where $p, \beta_{c}$ and $z$ are the momentum, velocity, and charge number of the incident particle, and $x / X_{0}$ is the thickness of the scattering medium in radiation lengths [3].

- positron annihilation is the interaction of a positron with an electron of the medium, resulting in the annihilation of the positron and electron, and the creation of two photons.

- ionization: occurs when an incident charged particle deposit energy in a medium in such a way that electrons are freed from atoms. It is modeled as a continuous energy loss of the incident particle when no secondary emerges from the medium, but for sufficient energy ionization results in the creation of $\delta$-rays, i.e. secondary electrons scattered by an incident electron (Möller process) or positron (Bhabha process). This is the predominant process by which low energy electrons (below $10 \mathrm{MeV}$ ) lose energy in matter.

- bremsstrahlung: is the production of photons by a charged particle that is accelerated in the field of atoms. It is the predominant process by which high energy electrons (above $10 \mathrm{MeV}$ ) lose energy in matter.

- photoelectric effect: refers to the emission of electrons from the surface of, generally, metals, in response to the absorption of an incident photon. This process is the main cause of interaction with matter for low energy photons.

- Compton scattering: a photon can be scattered by a free electron, which gains momentum in the process. The scattered photon wavelength differs from the initial one by:

$$
\lambda^{\prime}-\lambda=\frac{h}{m c}(1-\cos \theta)
$$

where $\theta$ is the angle between the incident and outgoing photon.

The photons produced by the above processes may be involved in further pair production. In summary, the expected processes are all electromagnetic in nature, and the complexity of the analysis stems from two sources, the stochastic muitiple scattering and the electromagnetic showering of the electron positron pair. The showering increases with the energy of the incident photon. 


\section{PROBABILISTIC EVENT ANALYSIS}

The methodology that we are developing works in principle as follows

- Run the simulator a large number of times, with the incident photon drawn from a uniform distribution. Retain those runs of the simulator where the same detector strips fired as in the event. Estimate quantities of interest (means etc. of the photon angles) from the runs retained.

Clearly the system is sufficiently complex that the number of runs is prohibitively large, and the description in this section is of a methodology that aims to only simulate those runs that will be retained.

Bayes theorem tells us how to update our beliefs when new data arrive, and how to use the distribution from one observation as a prior when analysing the next observation. Applied to one event of the LAT, we can impose a time-ordering on the data, and incorporate the microstrip firing information one detector layer at a time. The advantage of this sequential approach is that the distribution over $\theta$ (azimuth, elevation, energy) becomes gradually more peaked as the data is incorporated, making the search for the region of parameter space that has significant probability mass more straightforward $[4,5]$.

We adopt ideas from particle filtering, and represent the distribution over $\theta$ by a set of uniformly weighted random samples, which are called the "particles"[6, 7]. Each particle represents a possible event - it contains information about the direction and energy of the incident photon, and also "nuisance parameters" representing such things as where in the detector the pair production occured, the scattering information as the electron and positron pass through the instrument, etc. The latter parameters are not of primary interest, but are needed to compute the probability of an event.

Initially we simplify the system to that shown in figure 4 . It has been reduced to twodimensions, and we assume that the microstrip detectors are perfect - there are no missed detections, resulting in a zero-one likelihood - any simulated event which triggers the microstrips that actually fired has likelihood one, and any simulated event that either does not fire all the detectors, or fires extra detectors, has likelihood zero.

\section{Methodology}

The particles at layer $i$ are distributed as ${ }^{1} p\left(\theta_{1: i} \mid d_{1: i}\right)$, and to incorporate the data at layer $i+1$ requires updating this to $p\left(\theta_{1: i+1} \mid d_{1: i+1}\right)$, where $\theta_{1: i+1}=\left\{\theta_{1: i}, \theta_{i+1}\right\}, \theta_{i+1}$ being the new nuisance parameters (e.g. scattering angles) at this level.

$$
p\left(\theta_{1: i+1} \mid d_{1: i+1}\right) \propto p\left(d_{i+1} \mid \theta_{i+1}\right) p\left(\theta_{1: i} \mid d_{1: i}\right) p\left(\theta_{i+1}\right)
$$

$p\left(d_{i+1} \mid \theta_{i+1}\right)$ is the likelihood of observing the data at layer $i+1$, and $p\left(\theta_{i+1}\right)$ is the prior on the nuisance parameters, for example the distribution of the scattering angles defined

\footnotetext{
${ }^{1}$ We use the subscript $1: i$ to denote the set of variables at layers $1 \ldots i$.
} 


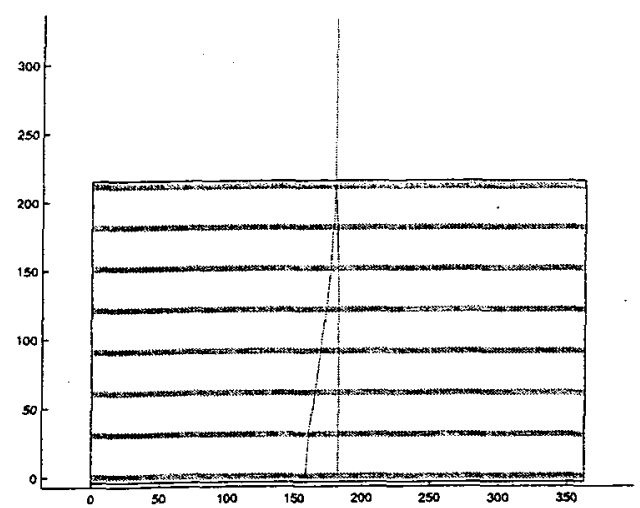

FIGURE 4. Simulation of the interaction of a $200 \mathrm{MeV}$ photon with the LAT.

by the physics of electrons passing through the material of the detector.

The standard manner to compute this update is to generate $\theta_{i+1}$ by sampling from $p\left(\theta_{i+1}\right)$, and then weight by the likelihood $p\left(\theta_{i+1} \mid d_{i+1}\right)$. However, sampling from $p\left(\theta_{i+1}\right)$ will result in most of the particles having trajectories that do not intersect the detectors that fired, and thus having likelihood zero ${ }^{2}$. Instead, we rewrite the update equation as

$$
p\left(\theta_{1: i+1} \mid d_{1: i+1}\right) \propto p\left(d_{i+1} \mid \theta_{i+1}\right) p\left(\theta_{1: i} \mid d_{1: i}\right) p\left(\theta_{i+1}\right) \frac{p_{I S}\left(\theta_{i+1}\right)}{p_{I S}\left(\theta_{i+1}\right)}
$$

where $p_{I S}\left(\theta_{i+1}\right)$ is an importance sampling distribution [8], that we choose to be concentrated around the values of $\theta_{i+1}$ which result in large likelihood values. The particles then have attached a weight given by $p\left(d_{i+1} \mid \theta_{i+1}\right) p\left(\theta_{i+1}\right) / p_{I S}\left(\theta_{i+1}\right)$ and the particles are resampled from the distribution defined by the weights, to give a set of samples that represent $p\left(\theta_{1: i+1} \mid d_{1: i+1}\right)$.

Note, however, that the elements of $\theta_{1: i+1}$ that are of primary interest, (the photon angle and energy), do not change as the data at subsequent layers is incorporated. This causes the number of particles that are distinct with respect t0 the incident photon angles and energy to be rapidly reduced, and the representation of the distribution to be poor. For this reason, after each new layer is incorporated, we preform a few Metropolis-Hastings updates [9] on all the elements of $\theta_{1: i+1}$. This leaves the distribution unchanged, but reintroduces diversity into the samples.

Consider the event shown in figure 4 . Initially the particles are uniformly distributed - the gamma ray may have come from any point in the sky, and entered the instrument at any $\mathrm{x}$-position. If we consider the first detector that fires as we move down the stack, then it is clear that the pair-production event must have occured in a small region above that microstrip, so that the electron and positron pass through the microstrip(s) that fired.

2 We could instead sample from the likelihood and weight by the prior. While this is possible for the simplified detector, this is likely to be infeasible for the actual instrument. 
We therefore construct the first importance sampling distribution to focus the position of the pair production event to be in this small region, and the angle of the incident photon to be in a small cone determined by the firings at this first level, and those at the next level - the size of this cone will depend on the energy of the photon, as that determines the distribution of the opening angle. At subsequent levels we construct an importance sampling distribution which puts large probability mass on the scattering angles that deflect the trajectories such that they intersect with the detectors that fired.

\section{SIMULATION RESULTS}

We present results from simulations of a simplification of the detector. Simulations in 2D are shown in figures 5 and 6 ; a 3D simulation is shown in figure 7 . The gamma conversion probabilities are those given in [3], as are the distributions of the energy split between the electron and the positron (see also figure 3 ). To simulate the multiple scattering, ionisation and bremsstrahlung processes, we used Geant4 [10] to simulate the interaction of an electron with a tungsten foil, and fit a gamma distribution to the resulting scattering angles for a wide range of electron energies. For the event analysis we assumed that the energy split is known - this is necessary in this simplification because we do not model the loss of energy by the electron and positron, so there is no information about the energy in the scattering angles along the electron and positron tracks.

Figure 5 shows a $200 \mathrm{MeV}$ gamma ray normally incident. The energy split is almost equal $(107: 93 \mathrm{MeV})$, and so the "opening angle" is almost symmetric. However, one of the tracks happens to undergo more significant scattering than the other. The histograms of the photon angle and position as data from subsequent layers are included is also shown. The distributions become clearly more peaked. Note, however, that because of the finite width of the detector strips, and the fact that the incident photon hit at the very left of the strip, the posterior distribution is biased away from the true values - moving the interaction point to the right and increasing the incidence angle slightly allows tracks that have smaller scattering angles, and are therefore more probable.

Figure 6 shows another $200 \mathrm{MeV}$ photon, where the energy split is much less equal $(154: 46 \mathrm{MeV})$, but where the opening angle is much smaller than the event in figure 5 . The low energy track undergoes much more scattering (the high energy track in this case is almost straight). Because of these characteristics of the event, the parameters of the actual event are very close to the modes of the distributions.

Figure 7 shows a $3 D$ simulation, and the estimates of the distributions of the incidence angles and the pair production position. These distributions also become more peaked as data at subsequent layers is incorporated. However, after four layers particle starvation became a problem, indicating that better importance sampling distributions need to be developed. 

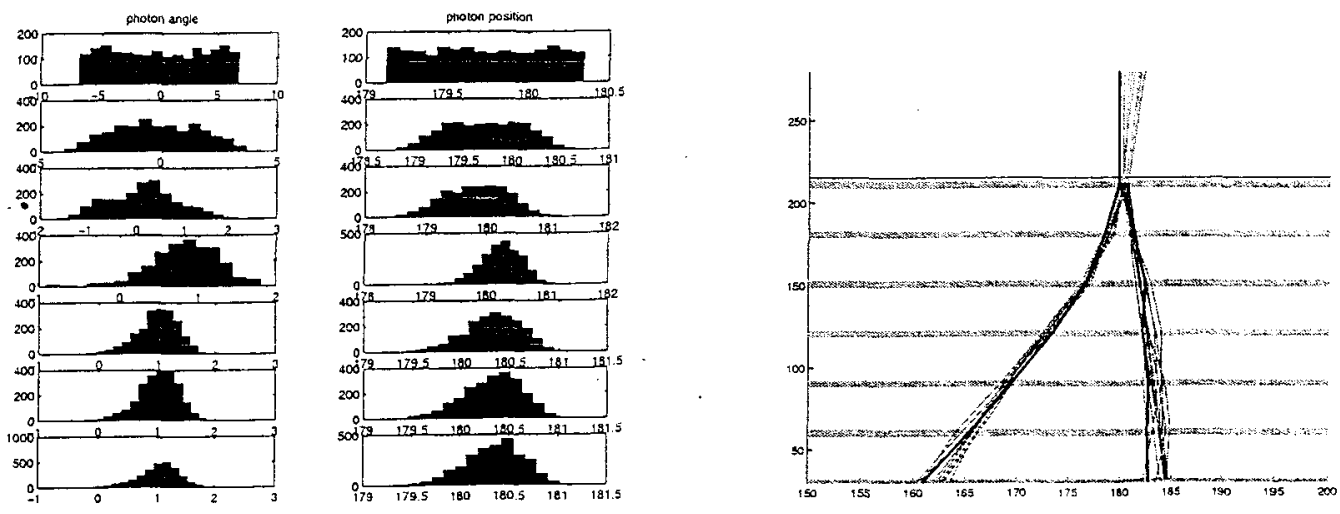

FIGURE 5. Estimated distribution of the incident photon angle and position as data from each layer is incorporated (left). The actual trajectory (bold) and a sample of the trajectories represented by the particles (right).
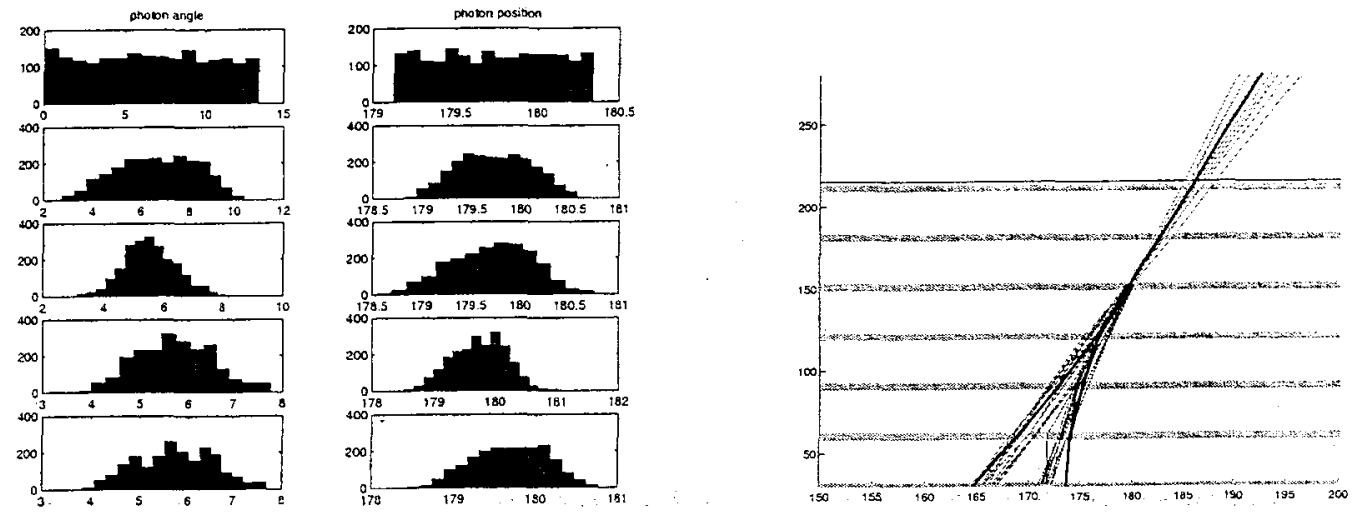

FIGURE 6. Estimated distribution of the incident photon angle and position as data from each layer is incorporated (left). The actual trajectory (bold) and a sample of the trajectories represented by the particles (right).

\section{DISCUSSION}

We have outlined an analysis methodology which we believe to be extremely well suited to the analysis of events from silicon microstrip type detectors such as those on the GLAST Large Area Telescope. We have shown through (admittedly very simplified) simulation the basic applicability of this methodology. Current and future work is devoted to incorporating more of the physics processes into the analysis (especially secondary particles, which have the potential to significantly complicate the analysis; and energy loss by the particles as they traverse the detector), and improving the likelihood model - it is necessary to incoiporate a better model of the firing of the microstrips, and include the fact that noise in the electronics may cause firings unrelated to the event itself. 

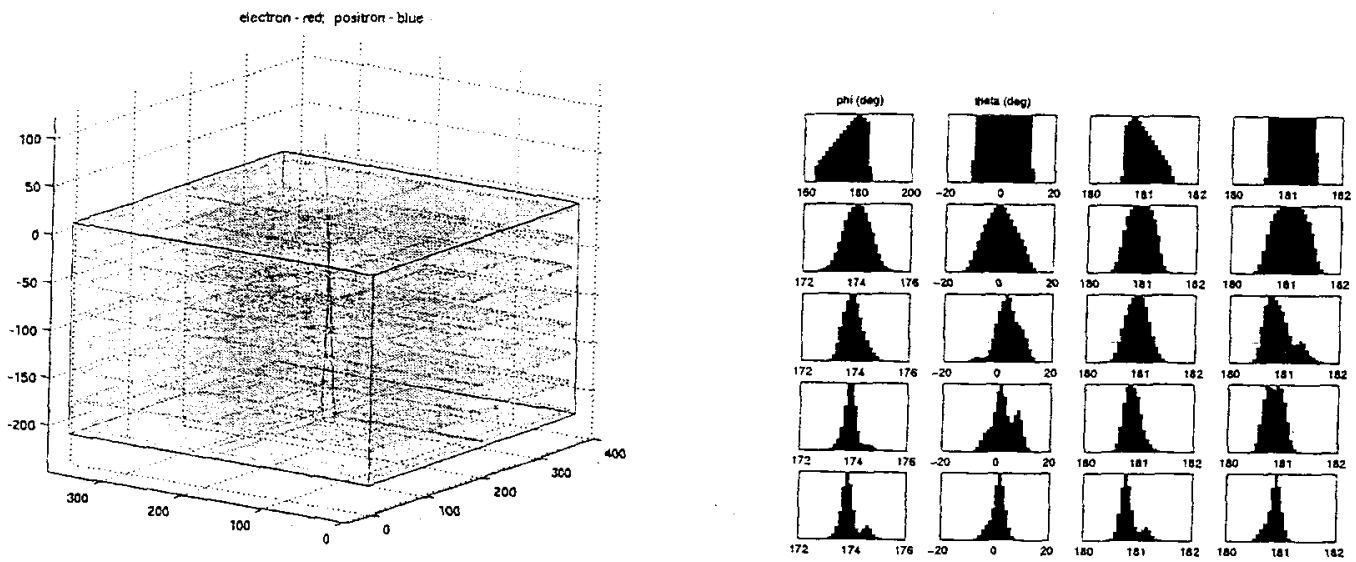

FIGURE 7. A simulated event for the simplified 3D detector (left). Estimated distributions of the azimuth and elevation angles, and the $(x, y)$ position of the pair production event (right).

\section{ACKNOWLEDGMENTS}

This work was funded in part by the NASA Ames Director's Discretionary Fund.

\section{REFERENCES}

1. GLAST Science Brochure, Tech. Rep. P-2000-9-107-GSFC, NASA-Goddard Space Flight Center (2001), URL http: //glast .gsfc.nasa.gov/resources/brochures/gsd/.

2. Boinee, P., et. al., "Gleam: the GLAST Large Area Telescope Simulation Framework," in Science with the New Generation of High Energy Gamma-ray Experiments, edited by S. Ciprini, A. De Angelis, P. Lubrano, and O. Mansutti, 2003.

3. Hagiwara, K., et. al., Phys. Rev. D., 66, 010001 (2002).

4. Smith, A., and Gelfand, A., The American Statistician, 46, no 2 (1992).

5. Chopin, N., Biometrika, 8, 195-201 (2002).

6. Gordon, N., Salmond, D., and Smith, A., IEE Proceedings-F, 140, 107-113 (1993).

7. Doucet, A., de Freitas, N., and Gordon, N., editors, Sequential Monte Carlo in Practice, Springer Verlag, 2001.

8. van de Merwe, R., Doucet, A., de Freitas, N., and Wan, E., "The Unscented Particle Filter," in Advances in Neural Information Processing Systems (NIPS13), edited by T. Leen, 505, 2000.

9. Gilks, W., et. al., editors, Markov Chain Monte Carlo in Practice, Chapman and Hall, London, 1996.

10. Geant4 physics reference manual; geant4 software reference manual (2002), http://wwwinfo. cern.ch/asd/geant4/G4UsersDocuments/Overview/htmi/index.html home page: http: //wwwinfo.cern.ch/asd/geant4/geant 4 .html. 